See discussions, stats, and author profiles for this publication at: https://www.researchgate.net/publication/227188870

\title{
A home e-health system for dependent people based on OSGI
}

Chapter · January 2009

DOI: 10.1007/978-1-4020-9823-9_9

\section{CITATIONS}

7

6 authors, including:

$$
\text { Ralf Seepold }
$$

Hochschule Konstanz Technik, Wirtschaft und Gestaltung Konstanz 173 PUBLICATIONS 464 CITATIONS

$$
\text { SEE PROFILE }
$$

Juan Antonio Alvarez-Garcia

Universidad de Sevilla

75 PUBLICATIONS 630 CITATIONS

SEE PROFILE
2. Natividad Martínez Madrid 176 PUBLICATIONS 476 CITATIONS

$$
\text { SEE PROFILE }
$$

2 Alejandro Fernández-Montes

E. Universidad de Sevilla 50 PUblications 305 Citations SEE PROFILE

Some of the authors of this publication are also working on these related projects:

Platforms View project

REACT: EneRgy efficiency and pErformAnCe of data centers by smart virTuallzation and deep learning event detection. View project 


\title{
Chapter 9
}

\section{A Home E-Health System for Dependent People Based on OSGI}

\author{
Jaime Martín ${ }^{1}$, Ralf Seepold ${ }^{1}$, Natividad Martínez Madrid ${ }^{1}$, Juan Antonio \\ Álvarez ${ }^{2}$, Alejandro Fernández-Montes ${ }^{2}$ and Juan Antonio Ortega ${ }^{2}$ \\ ${ }^{I}$ Universidad Carlos III de Madrid, Spain; \\ ${ }^{2}$ Universidad de Sevilla, Spain \\ natividad.martinez@uc3m.es,ralf.seepold@uc3m.es,jortega@us.es,alejandro.fdez@gmail.com, \\ jaalvarez@us.es
}

\begin{abstract}
This chapter presents a e-health system for dependent people installed in a home environment. After reviewing the state of art in e-health applications and technologies several limitations have been detected because many solutions are proprietary and lack interoperability. The developed home e-health system provides an architecture capable to integrate different telecare services in a smart home gateway hardware independent from the application layer. We propose a rule system to define users' behavior and monitor relevant events. Two example systems have been implemented to monitor patients. A data model for the e-health platform is described as well.
\end{abstract}

Keywords e-health, telemedicine, telecare, dependent people, orientation, home automation, OSGi, HL7.

\subsection{Introduction}

The World Health Organization defines the term e-health as the relations between institutions, public health, e-learning, remote monitoring, telephone assistance, domiciliary care and any other system of remote medicine care. Telecare is the part of e-health that offers remote care of dependent (elderly or disabled people), providing the care and reassurance needed to allow them to live in their own homes.

Integration of healthcare key actors is required to offer quality in service but e-health systems often lack adequate interoperability or integration of social aspects; the results is a slow down in acceptance and usage of these 
systems. Integrating Information and Communication Technologies (ICT) (like for example telehomecare) in care, living and wellness is a citizens demand that it should be provide at affordable cost [1].

An example of e-health services integration challenge is to achieve communicate to dependent people with relatives and medical people, integrating movement monitorization and orientation together with electronic medical record transmission. Consequently, multimedia communication and tracking people technologies should be seamlessly integrated in the e-health service. Residential Gateways (RGW) enable telecom companies to provide applications in a home environment via a platform to manage several services remotely. The OSGi (formerly known as the Open Services Gateway initiative, www.osgi.org) specification provides an architecture for remote control of a platform. OSGi also includes support for the whole life-cycle of services (e.g. start, stop, etc.). This chapter proposes a platform for a home e-health system based in OSGi that provides capabilities to integrate different telecare, telemedicine and orientation support services for dependent people in the same RGW.

In Section 9.2, the state of the art of telemedicine, telecare and electronic health record standards is reviewed. Section 9.3 presents the new proposal by describing two patient monitorization examples, a production system, use cases and the data model. Finally, Section 9.4 sums up the results so far and describes some future work.

\subsection{State of Art}

\subsubsection{Related Research on Telecare and Telemedicine}

Several residential telecare and telemedicine platforms approaches are presented in literature. Bobbie [2] describes an electronic-prescription system for home-based telemedicine using the OSGi framework. This article describes a health-prescription application running on a smart card that communicates with a Personal Digital Assistant (PDA). It uses OSGi as a central coordinating point between different devices. The OSGi environment is aimed to allow intercommunication between the card reader, the patient's PDA application and other devices. However, it lacks a detailed description about how the system is implemented and how security needs are reflected in the application implementation that are specific in a medical environment.

The Service-Oriented Agent Architecture described in another approach [3] enables healthcare services providers to support telecardiology services on demand. It proposes a runtime unit of telemedicine agents to permit 
services to be managed remotely. The system consists of an agent unit, which includes a vital signals acquisition module; it can acquire ECG (electrocardiogram) data and forward ECG data to a medical service center. It uses Web Services to communicate to all services via XML in multiple platforms but it does not mention any EHR standard.

Some Spanish research projects have presented a generic architecture to e-health system based on middleware components [4]. The goal is to provide a service platform for any kind of e-health application. These generic architectures should allow a quick development of new services using the existing infrastructure. Currently, the HL7 standard is ruled out because it lacks interoperability with other systems and EN 13606 is proposed to represent and to transmit clinic information. Currently, a proposal of an IEEE 11073 platform for healthcare tele-monitoring has been published [5]. This work identifies a set of use cases relevant for a personal monitoring scenario and it identifies related features and functionalities.

\subsubsection{E-Health Technologies}

Health patient data must be transmitted and saved in a standard format supported by all involved systems. An electronic health record (EHR) refers to an individual patient's medical record in digital format. An EHR standards comparative study [6] describes HL 7 and EN 13606 standards. To transmit medical information between devices IEEE 11073 has been developed. A brief overview of these technologies is presented in the following three sub-sections.

\subsubsection{HL7}

Health Level Seven (HL7) [7,8] is a widely applied protocol to exchange clinical data. Several versions are been developed by the HL7 organization, part of American National Standard Institution and founded in 1987. Version 3 is beyond the scope of this chapter because it is a complex standard and there is no a stable version available [9].

The HL7 refers to seventh OSI layer (application) although it also specifies a layer 6 presentation protocol made up of its own abstract message format and encoding rules. Concerning the lower layers, like session and transport services, it is rather vague because the HL7 authors' intention was to support a wide variety of systems. The underlying HL7 operational model is a client-server system. For example, when a patient is admitted to a hospital, the admission system will propagate HL7 admission messages to an appropriate subsystem. An HL7 message always contains all the information 
required to complete a transaction and it is encoded in HL7 rules. Essentially, all information is transmitted in ASCII plain text. The standard allows defining site-specific extensions segments, like message extensions to exchange data with an appointment system. However, the use of these extensions can prompt serious interoperability problems. Moreover, HL7 lacks a specific methodology to generate messages and it is not clear how the structural relations between fields are defined.

\subsubsection{EN 13606}

Health informatics - Electronic Health Record Communication standard (EN 13606) is a European official standard of CEN (European Committee for Standardization) and an approved ISO standard. The overall goal is define a rigorous and stable information architecture for communicating part or all of the EHR of a patient. It is based on the HL7 RIM (Reference Information Model) from HL7 v3, a set of data type definitions harmonized between HL7 and CEN, the EHR Domain Information Model (DMIM) and a bunch of RMIMs dedicated to certain structures and functionalities.

EN 13606 is flexible to represent the information structures transmitted thanks to the archetypes, a knowledge representation of the clinic information domain. Moreover, it is robust with respect to changes in the specifications because changes in the archetypes do not provoke a change in the underlying system.

The openEHR framework (www.openehr.org) is compliant to the EN 13606 and it is used in commercial systems throughout the world.

\subsubsection{ISO/IEEE 11073}

A brief description of novel standards ISO/IEEE 11073, often also referred to Medical Information Bus (MIB), or x73 standards, for personal telemedicine systems interoperability can be found in [10]. The goal is to enable medical devices to interconnect and interoperate with other medical devices. These standards cover the upper OSI layers and use well-known IEEE standards like Bluetooth (802.15.1) or WLAN (802.11) in lower layers. Part of $x 73$ standards focus on point-of-care medical devices communication which are mainly designed for acute monitoring and treatment application in a particular diagnostic, bed or treatment area in the hospital domain like Intensive Care Unit (ICU). Several of x73 standards series are currently drafts and new projects are under development; first prototype implementations are available in industry. 
The standard is based on an object-oriented system management paradigm. A numeric code set identifies every item that is communicating. This is more efficient than HL7 because it uses binary instead of plain text data. The key objectives for clinical domain applications addressed by the standard are real-time plug \& play interoperability and frequent network reconfiguration. Special attention has been paid to reduce implementation complexity and computational burden of devices. For wireless devices, transmission power and transmission time could be reduced.

\subsection{E-Health Service Proposal for Dependent People}

\subsubsection{Overview}

Our proposal attempts to integrate several smart home services to provide a scalable and interoperability e-health solution. The system is divided into three basic subsystems: domotic, multimedia and e-health subsystem. In the domotic subsystem different devices can co-exist in each subsystem connected by wire or wireless to a residential gateway (RGW) based on an embedded OSGi framework. Blood-pressure monitor and a pair of scales are examples of integrated devices in the medical network.

A domotic environment based on a Lonwork ${ }^{a}$ network typically includes sensors and actuators, for example light sensors or blind motor. A Lonworks platform provides a reliable and open protocol accepted as a standard for control networking. As we see in data model, a RGW can obtain some useful environment variables from automation home network so when a relevant event occurs, an alert or alarm is reported in the RGW. This event can be sent to relatives or medical people. The multimedia network typically includes a television, an IP camera or a webcam with microphone, necessary for dependent person to communicate. The RGW is able to physically interconnect all required networks and devices, and to host different services which can be managed remotely by the e-health or access provider. Multimedia services, like SIP audio/videoconference are provided to communicate during the medical tele-visit (cf. Fig. 9.1).

\footnotetext{
${ }^{a}$ http://en.wikipedia.org/wiki/LonWorks
} 


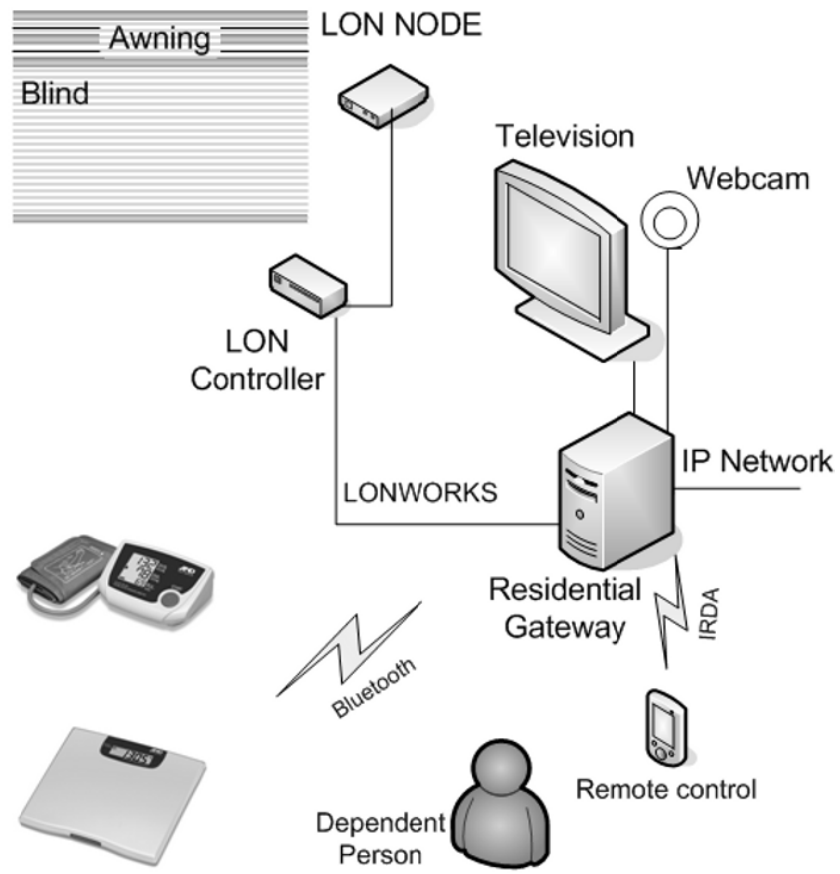

Fig. 9.1 Smart Home overview, with automation, multimedia and medical devices

\subsubsection{Movement Monitorization and Orientation}

In this section we propose two systems to monitor patients. The first one monitors outdoors trips and the second one indoor activity.

The first uses an inference engine that does not require users to explicitly provide information about the start or end points of their journeys; instead this information is learned from users' past behavior. The initial requirement is the tracking of frequent trips of the monitored users. In order to test our system, a user carried a GPS logger with him continuously for 24 hours a day for 30 days. GPS data was filtered and segmented to obtain the user's trips. Then the GPS trips with spatial and temporal data were clustered in routes (only spatial information) using a dendrogram clustering tested by Froehlich and Krumm at Microsoft [11]. The routes were used to make a supervised learning with new trips. Figure 9.2 shows the routes obtained from one user. 
Using similarities that measure point to point distances between a new trip and the existing routes and a very early classification was done about the route and the user's goal when the trip is beginning.

The trained model correctly identifies more common places and whole routes, allowing the detection of lost or disorientation situations in open areas.

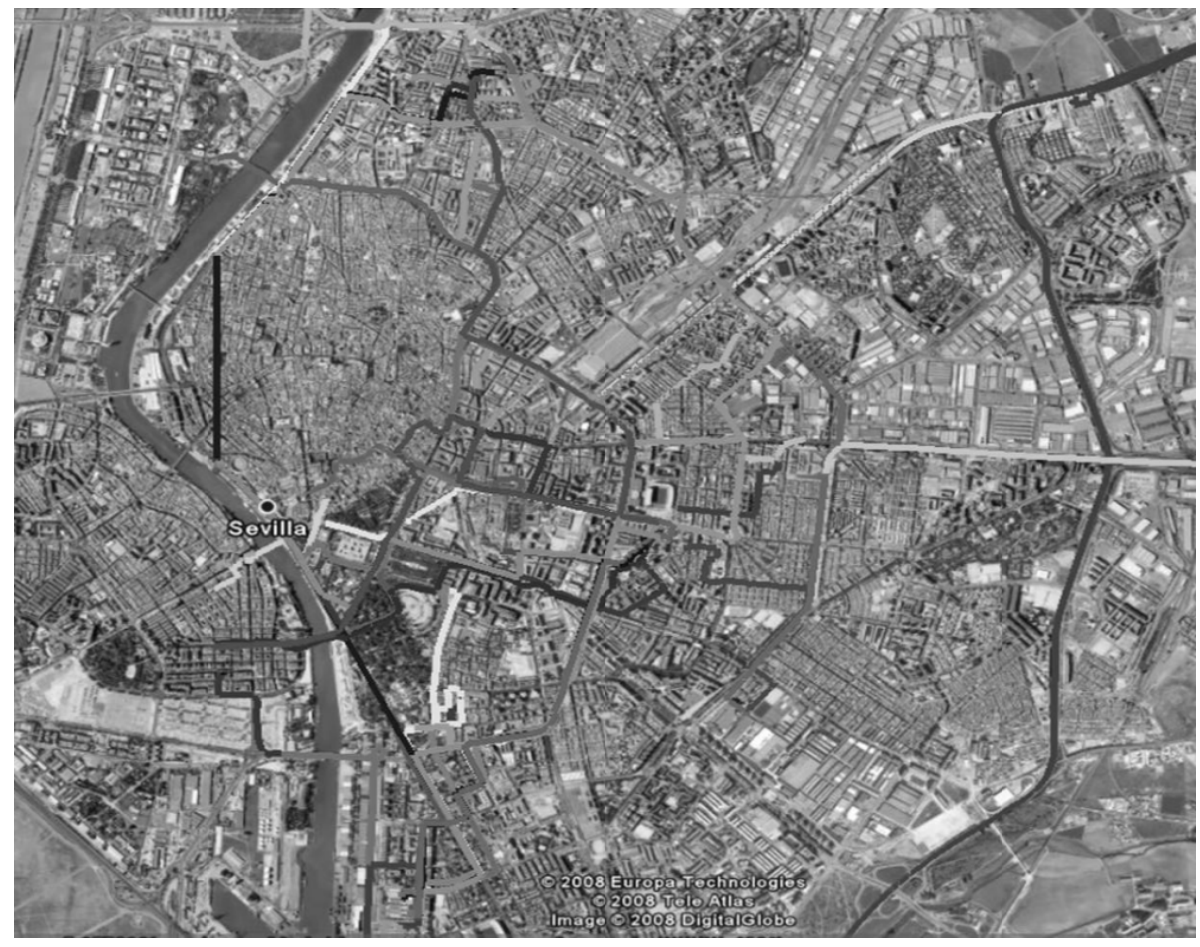

Fig. 9.2 A example of GPS tracking

When a set of GPS locations are received and all of them are located out of the security area of the routes, different policies could be selected to prevent dangerous situations:

- If the user is a dependent person or his abilities handling a smartphone are low, the first option is to send an automatically generated SMS send to previously selected people showing the exact location of the user. If an Internet connection is available, the text of the SMS is an address obtained from a Web Service that provides the close address to a latitude-longitude pair (reverse geocoding). If not, the text is only the latitude-longitude pair. 
- Another policy is a reorientation service that helps the user to reach the closest point from a frequent route and then guide him to a frequent place (he could choose one from photographs of the common ones). In a previous work [13] we attempted to design a reorientation application to Alzheimer patients but the lack of families that allow a relative that suffers that disease to go out alone and the difficulties to manage a device like the HTC P3300 by this kind of people in a crisis situation make us change the interaction and the potential users. In [14] a similar application was explained but the users' audience was different (mild cognitive disabilities).

Due to the fact that users usually are indoors, the system proposed was not complete to monitor and help them. To improve the usefulness, a second system has been designed using the smartphone and a device called Alive Heart Monitor [15] (cf. Fig. 9.3) composed by an ECG and a tri-axial accelerometer. The weight (55gr with battery), measurements $(90 \times 40 \times 16$ $\mathrm{mm}$ ), and operating live without change the battery and sending the data wireless ( 48 hours) make it perfect to be worn by the user. The only problem is that two liquid gel electrodes must be placed on the user's chest to obtain high quality ECG signal. This is normally bothering the user for a long term monitoring.

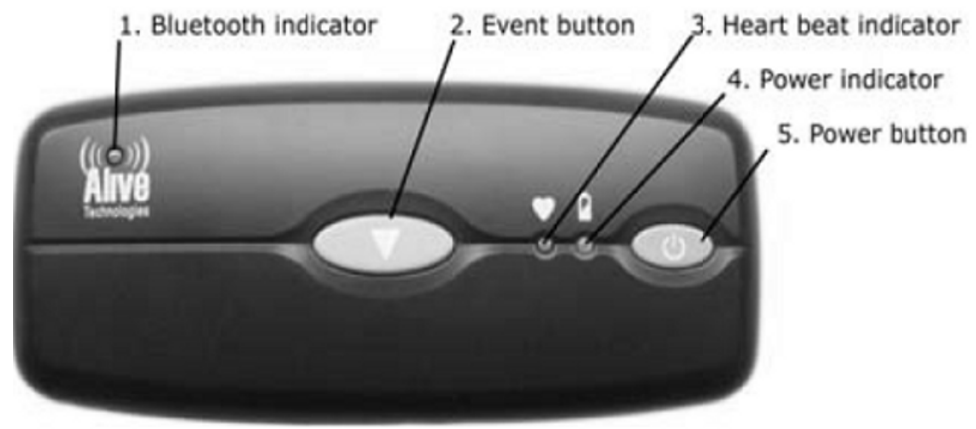

Fig. 9.3 Health monitor

The ECG data helps to detects potential heart illness and the heart rate. The accelerometer data depending on the position of the device helps to classify the activities of the user (running, walking, standing up, sitting down) and other risky patterns like falls (a big acceleration followed by a sudden stop and a body change of orientation). 
A rule-alert system was designed to send automatically a SMS to the relatives; an OSGi-Server stores vital constants to be queried by authorized users.

\subsubsection{Production System}

A rule-based system to control and monitorize the user's behavior is presented here. This is a production system formed by a facts base and rules base as showed in Fig. 9.4. This has been implemented as a rule engine and scripting environment ${ }^{\mathrm{b}}$ written in Java language by Jess.

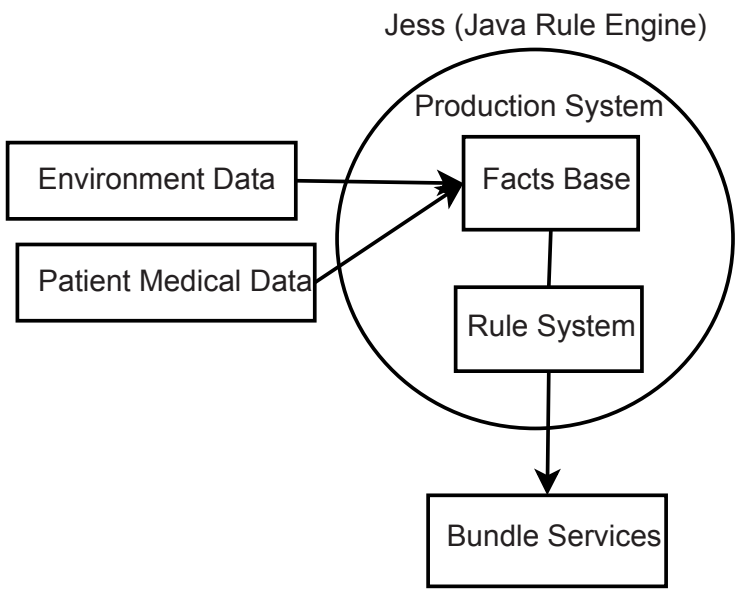

Fig. 9.4 Production System for e-Health system

In Table 9.1 we show an example of Rules Base for our e-Health platform with health and automatic rules based on a health monitor and other sensors.

Table 9.1 Example of rules base for e-health system

\begin{tabular}{ll}
\hline Condition & Action \\
\hline High Blood Pressure Rate & Call to health professionals \\
High Blood Pressure Rate & Call to relatives \\
Low Light Level & Rise up blind \\
\hline
\end{tabular}

In certain cases, we need a priority range for these rules because it is possible that several rules apply at the same time. Priority and urgency

\footnotetext{
${ }^{\mathrm{b}}$ http://herzberg.ca.sandia.gov
} 
issues for a mobile care system are treated in [16]. Alert mechanisms in actions should have a priority level because some situations could be critical or very critical and they need a faster response. For example, if the patient has a lot of pain he can contact the assistant before relatives are informed. The assistant can then decided if an ambulance is required to attend the patient.

\subsubsection{Use Cases}

We can identify some important use case of the platform. A general administrator controls the RGW but can appear that there are different administrators for each subsystem like an e-health admin, which is allowed to configure e-health devices only. A solution to separate different RGW administration by virtualization is described in [17]. Every service offered by a device is part of a scene, i.e. a set of pre-established services by the admin. Users without administration permission, like relatives, friends, assistants or dependent person can directly customize the devices to adapt them according to their preferences. For example, a relative of a dependent person can set the hours that a blind is open to allow illuminate the room during the periodic blood pressure check.

Telecare with assistant people of the patient/dependent person can be another use case, like it is shown in Fig. 9.5.

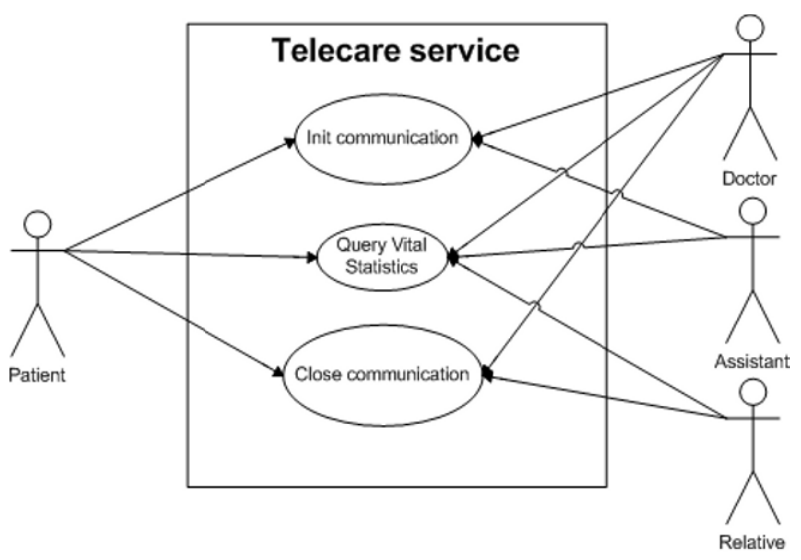

Fig. 9.5 Telecare use case

This is often organized without considering a communication with relatives and friends. The problem is that the patient usually prefers to contact first of all his relatives and friends if they need anything. According 
to several studies, dependent people are reluctant to use many health care services because they do not personally know the operator or the contact person in the service centre. So an objective of this work is to integrate relatives and friends into the healthcare service provision, and thus increase the usability of the system. For example, in this use case the assistant initiates a SIP video call with the patient and with a relative. He checks remotely the vital statistics like weight or blood pressure thanks to health care devices at the patient home. The wireless devices are connected via $\times 73$ standards (or proprietary protocols) to RGW which recovers the medical information, processes data and saves them in HL7 format. Then, the data can be processed by the medical information system and displayed to the doctor in his computer.

\subsubsection{Data Model}

The data model designed is divided into the user management on one side and the device management on the other side. An overview of data model is shown in Fig. 9.6.

A generic User entity saves basic data, like name, surname, address, etc. Defined attributes in this schema should be compatible with HL7 PID (Patient Identifier Segment) fields. For example, Spanish second surname must be matched in Mother's Maiden Name (XPN) following the HL7 Spain recommendations (www.hl7spain.org). Relative, Assistant, Doctor and Administrator are entities which have different attributes and different roles according to their permissions. A role defines a permission to access to data or devices. In this manner, the user type definition is separated from privilege definition. The administrator user has total control over the full system in case he is the RGW admin or only partially in case he is member of another admin type, like the e-health admin. Automation, multimedia and medical devices have a link address and/or IP address, status, service list and several configuration variables. The RGW can save some parameters of a Lonworks node like for example its ID or its network variables to monitor the status of patient and his environment.

The doctor is allowed to access basic and medical data of the assigned patients. The assistant entity can represent a nurse or a social assistant. Information about medical visits, like diagnostic, reason and date, is saved in Visit entity. If a doctor orders a treatment, important dates and status are saved in Treatment. There is medicine administration sometimes, so frequency, medicine name and comments can be annotated in Medicine. A user (doctor, assistant or patient) can add remainders associated to a treatment, like exercises or medical administration remainders. 


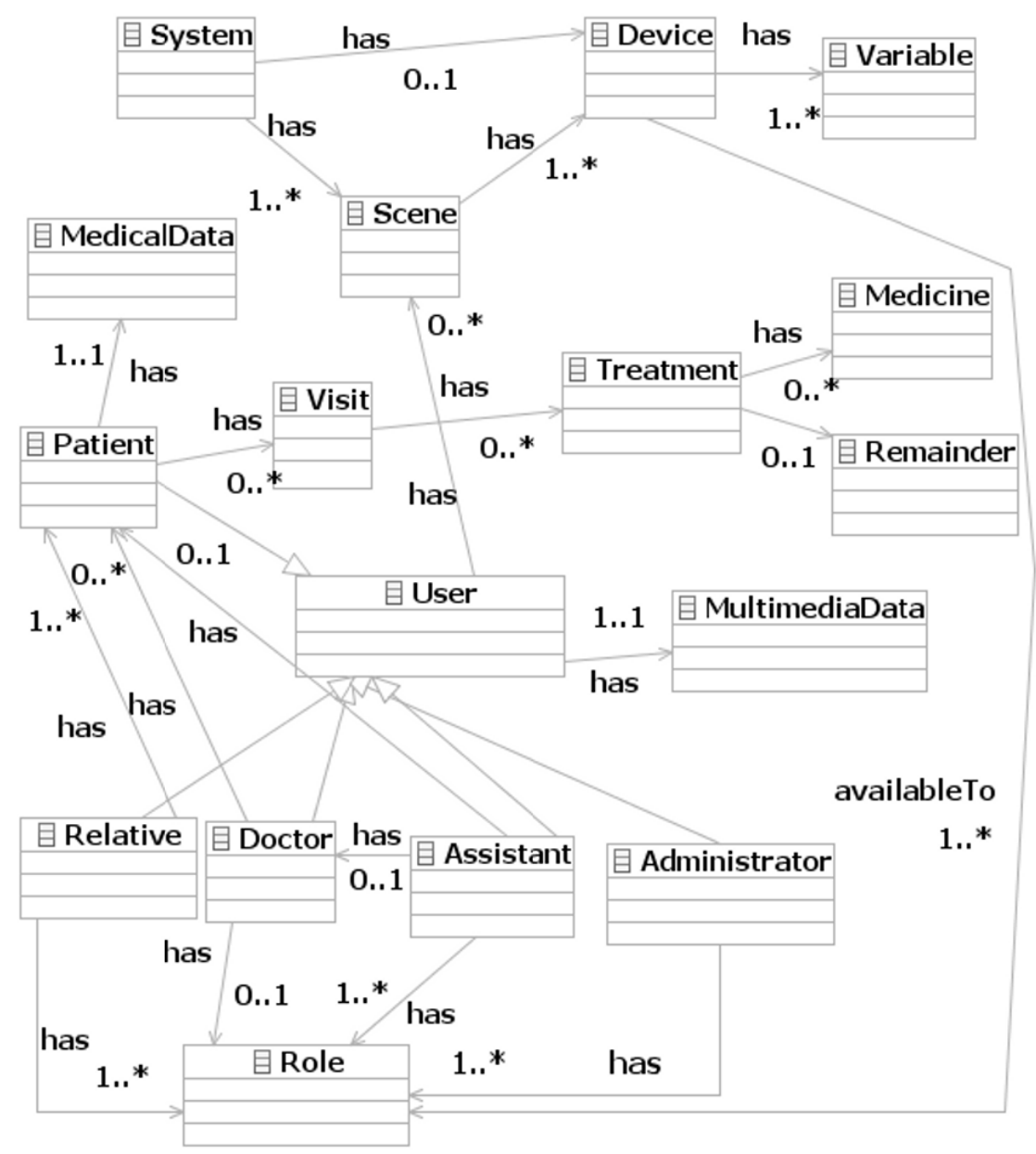

Fig. 9.6 Platform Data Model

\subsection{Conclusions and Future Work}

A new e-health system architecture has been presented that tries to integrate all relevant members into a common scenario. The dependent person, the medical personal and relatives (or friends) are integrated as members of the platform. Each entity has individual duties following the general objective to enhance the quality of life for the dependent person. Besides this important goal, the technical infrastructure has been developed to seamlessly integrate technical devices. Existing but also extended versions of standards are used 
and proposed in order to increase efficiency and usability in realistic scenarios. Future work will provide a basic implementation and test of use cases..

Acknowledgements This research is supported by the MEC I+D project InCare-FAMENET. Ref: TSI2006-13390-C02-02.

\section{References}

1. EHTEL Force. Sustainable Telemedicine Task. Sustainable Telemedicine: paradigms for future-proof healthcare. A brief Paper. European Health Telematics Association (EHTEL), 2008.

2. P.O. Bobbie, S.H. Ramisetty, A. Yussiff, and S. Pujari. Designing an Embedded Electronic-Prescription Application for Home-Based Telemedicine Using OSGi Framework. In Embedded Systems and Applications, H.R. Arabnia and L.T. Yang, eds., 16-21, CSREA Press, 2003.

3. Y. Chen, and C. Huang. A Service-Oriented Agent Architecture to Support Telecardiology Services on Demand. Journal of Medical and Biological Engineering 25(2), 2005.

4. P. de Toledo, A. Muñoz, J.A. Maldonado, E. Hernando, R. Somolinos, P. Crespo, E. Gómez, F. del Pozo, M. Robles, and J.A. Fragua, Arquitectura genérica para sistemas de e-salud basada en componentes middleware. In Libro de Actas del XXIII Congreso Anual de la Sociedad Española de Ingeniería Biomédica (CASEIB’05), 29-32, 2005.

5. M. Galarraga, I. Martinez, L. Serrano, P. de Toledo, J. Escayolan, J. Fernandez, S. Jimenez-Fernandez, S. Led, M. Martinez-Espronceda, E. Viruete, and J. Garcia. Proposal of an ISO/IEEE11073 Platform for Healthcare Telemonitoring: Plug-and-Play Solution with new Use Cases. In Engineering in Medicine and Biology Society, 2007. EMBS 2007. 29th Annual International Conference of the IEEE, 6709-6712, 2007.

6. B. Blobel, and P. Pharow. EHR Standards-A Comparative Study. In Medical And Care Compunetics 3, ed. Lodewijk Bos, L. Roa, K. Yogesan, et al. IOS Press, 2006.

7. W. Hammond. Health Level 7: A protocol for the interchange of healthcare data. In Progress in Standardization, In Health Care Informatics, Georges J.E. De Moor, C. McDonald, and J.N. Van Goor, eds., Amsterdam: IOS Press, 1993.

8. A. Hutchison, M. Kaiserswerth, M. Moser and A. Schade., Electronic data interchange for health care. Communications Magazine, IEEE 34: 28-34, 1996.

9. B. Smith, and W. Ceusters. HL7 RIM: An Incoherent Standard. En Studies in Health Technology and Informatics. Ubiquity: Technologies for Better Health, In Aging Societies - Proceedings of MIE2006, A. Hasman, R. Haux, J. van der Lei, and F.H. Roger France, eds., 124:133-138. Amsterdam: IOS Press, 2006.

10. L. Schmitt, L. Schmitt, T. Falck, T. Falck, F. Wartena, and D. Simons. Novel ISO/IEEE 11073 Standards for Personal Telehealth Systems Interoperability. In High Confidence Medical Devices, Software, and Systems and Medical Device Plug-and-Play Interoperability, 2007. HCMDSS-MDPnP. Joint Workshop on, 146-148, 2007.

11. I. Martinez Ruiz, M. Galarraga, L. Serrano, P. de Toledo, S. Jiménez-Fernández, J. Escayola, E.A. Viruete, J. Fernández, and J. García. Enhanced Solutions for 
Healthcare Telemonitoring in Ambient Assisted Living based on ISO/IEEE11073 standard. Methods of Information in Medicine Special Topic Issue: Smart Homes and Ambient Assisted Living in an Aging Society. pp. pte (ISSN: 0026-1270. IF2005: 1.235), 2007.

12. J. Froehlich, and J. Krumm. Route Prediction from Trip Observations, In Society of Automotive Engineers (SAE) 2008 World Congress, 2008.

13. A. Fernández-Montes, J.A. Álvarez, J.A. Ortega, N. Martínez Madrid, and R. Seepold. An Orientation Service for Dependent People Based on an Open Service Architecture, HCI and Usability for Medicine and Health Care, Springer LNCS, Volume 4799, ISSN 0302-9743, 2007.

14. D. Patterson, and L. Liao. 2004. Opportunity Knocks: A system to provide cognitive assistance with transportation services. Proceedings of Ubicomp 2004.

15. Alive Technologies, http://www.alivetec.com ,2008.

16. R. Lee, K. Chen, C. Hsiao, and C. Tseng. A Mobile Care System With Alert Mechanism. Information Technology in Biomedicine. In IEEE Transactions on 11(5), (September): 507-517. doi:10.1109/TITB.2006.888701, 2007.

17. M. Ibañez, N. Martínez Madrid, and R. Seepold. Virtualization of Residential Gateways. In Proceedings of the Fifth International Workshop on Intelligent Solutions in Embedded Systems (WISES07, ed. Ralf Seepold, Natividad Martínez Madrid and Markus Kucera, 115-126. Leganés (Spain): Universidad Carlos III de Madrid, 2007. 